\title{
A retrospective analysis of hyperthermic intraperitoneal chemotherapy for gastric cancer with peritoneal metastasis
}

\author{
MEIQIN YUAN $^{1}$, ZENG WANG $^{2}$, GUINV HU $^{3}$, YUNSHAN YANG $^{1}$, \\ WANGXIA LV ${ }^{1}$, FANGXIAO LU ${ }^{4}$ and HAIJUN ZHONG ${ }^{1}$ \\ Departments of ${ }^{1}$ Chemotherapy and ${ }^{2}$ Pharmacy, Zhejiang Cancer Hospital, Hangzhou, Zhejiang 310022; \\ ${ }^{3}$ Department of Surgical Oncology, Dongyang People's Hospital, Dongyang, Zhejiang 322100; \\ ${ }^{4}$ Department of Radiology, Zhejiang Cancer Hospital, Hangzhou, Zhejiang 310022, P.R. China
}

Received January 11, 2016; Accepted May 25, 2016

DOI: $10.3892 / \mathrm{mco} .2016 .918$

\begin{abstract}
Peritoneal metastasis (PM) is a poor prognostic factor in patients with gastric cancer. The aim of this study was to evaluate the efficacy and safety of hyperthermic intraperitoneal chemotherapy (HIPEC) in patients with advanced gastric cancer with PM by retrospective analysis. A total of 54 gastric cancer patients with positive ascitic fluid cytology were included in this study: 23 patients were treated with systemic chemotherapy combined with HIPEC (HIPEC+ group) and 31 received systemic chemotherapy alone (HIPEC- group). The patients were divided into 4 categories according to the changes of ascites, namely disappear, decrease, stable and increase. The disappear + decrease rate in the HIPEC+ group was $82.60 \%$, which was statistically significantly superior to that of the HIPEC- group (54.80\%). The disappear + decrease + stable rate was $95.70 \%$ in the HIPEC+ group and $74.20 \%$ in the HIPEC- group, but the difference was not statistically significant. In 33 patients with complete survival data, including 12 from the HIPEC+ and 21 from the HIPEC- group, the median progression-free survival was 164 and 129 days, respectively, and the median overall survival (OS) was 494 and 223 days, respectively. In patients with ascites disappear/decrease/stable, the OS appeared to be better compared with that in patients with ascites increase, but the difference was not statistically significant. Further analysis revealed that patients with controlled disease (complete response + partial response + stable disease) may have a better OS compared with patients with progressive disease, with a statistically significant difference. The toxicities were well tolerated in both groups. Therefore, HIPEC was found to improve survival in advanced gastric cancer patients with PM, but the difference was not statistically significant, which
\end{abstract}

Correspondence to: Dr Haijun Zhong, Department of Chemotherapy, Zhejiang Cancer Hospital, 38 Guangji Road, Hangzhou, Zhejiang 310022, P.R. China

E-mail: zhj1712@aliyun.com

Key words: hyperthermic intraperitoneal chemotherapy, gastric cancer, peritoneal metastasis may be attributed to the small number of cases. Further studies with larger samples are required to confirm our data.

\section{Introduction}

Peritoneal metastasis (PM) is the most common metastatic pattern in gastric cancer (GC) and has an extremely poor prognosis $(1,2)$, with a median survival of 3-6 months without treatment (3). PM may lead to refractory ascites, intestinal obstruction, cachexia and eventually death. Thus far, PM is considered as stage IV disease, and treatment is non-curative.

There is currently no established consensus to direct treatment for GC patients with positive peritoneal cytology. Intraperitoneal chemotherapy (IPC) has been proposed as a treatment option, which may improve survival in GC patients patients with PM (4-8). IPC possesses a theoretical advantage over the systemic route by delivering high drug concentrations directly to the peritoneal cavity, with reduced systemic toxicity (9-11). In addition, high drug concentrations are achieved in the portal vein (12). This may be important, as the liver is a common metastatic site (13). Recently, hyperthermia has been developed as an anticancer therapy and has been shown to exert a direct cytotoxic effect on tumor cells in the peritoneal cavity in conjunction with certain chemotherapeutic agents (14). Zhao et al reported that whole-body hyperthermia combined with hyperthermic intraperitoneal chemotherapy (HIPEC) is an effective treatment for patients with advanced gastric malignancies (15). A meta-analysis by Sun et al demonstrated that HIPEC may improve the overall survival (OS) rate in patients who undergo resection for advanced GC, and help to prevent peritoneal local recurrence among GC patients with serosal invasion (16). HIPEC has also been suggested to be useful in treating advanced peritoneal metastatic cancer, and significantly prolonged survival following tumor resection $(17,18)$.

The aim of this study was to evaluate the efficacy and safety of HIPEC in advanced GC patients with peritoneal dissemination by retrospective analysis.

\section{Patients and methods}

Patients. The clinical records of 54 patients who were primarily diagnosed as GC with PM at the Zhejiang Cancer Hospital 
Table I. Baseline characteristics of HIPEC+ and HIPEC- patients.

\begin{tabular}{|c|c|c|c|c|}
\hline \multirow[b]{2}{*}{ Characteristics } & \multicolumn{2}{|c|}{ HIPEC+ group } & \multicolumn{2}{|c|}{ HIPEC- group } \\
\hline & $\begin{array}{c}\text { All } \\
(\mathrm{n}=23)\end{array}$ & $\begin{array}{c}\text { Complete survival } \\
\text { data }(\mathrm{n}=12)\end{array}$ & $\begin{array}{c}\text { All } \\
(\mathrm{n}=31)\end{array}$ & $\begin{array}{c}\text { Complete survival } \\
\text { data }(\mathrm{n}=21)\end{array}$ \\
\hline \multicolumn{5}{|l|}{ Gender, $\mathrm{n}$} \\
\hline Male & 7 & 5 & 11 & 7 \\
\hline Female & 16 & 7 & 20 & 14 \\
\hline \multicolumn{5}{|l|}{ Age, years } \\
\hline Median & 43 & 44.5 & 53 & 52 \\
\hline Range & $18-68$ & $31-68$ & $24-77$ & $24-66$ \\
\hline \multicolumn{5}{|l|}{ Primary site, $\mathrm{n}$} \\
\hline Esophagogastric junction & 1 & 1 & 1 & 0 \\
\hline Gastric body & 9 & 5 & 14 & 10 \\
\hline Gastric antrum & 10 & 5 & 9 & 6 \\
\hline Diffuse gastric lesions & 3 & 1 & 3 & 2 \\
\hline Unkown & 0 & 0 & 4 & 3 \\
\hline \multicolumn{5}{|l|}{ Pathology, n } \\
\hline Well-differentiated & 0 & 0 & 0 & 0 \\
\hline Moderately differentiated & 0 & 0 & 3 & 2 \\
\hline Poorly differentiated & 18 & 8 & 27 & 18 \\
\hline Differentiation unknown & 5 & 4 & 1 & 1 \\
\hline \multicolumn{5}{|l|}{ Radical surgery, $\mathrm{n}$} \\
\hline Yes & 4 & 4 & 8 & 5 \\
\hline No & 19 & 8 & 23 & 16 \\
\hline
\end{tabular}

HIPEC, hyperthermic intraperitoneal chemotherapy.

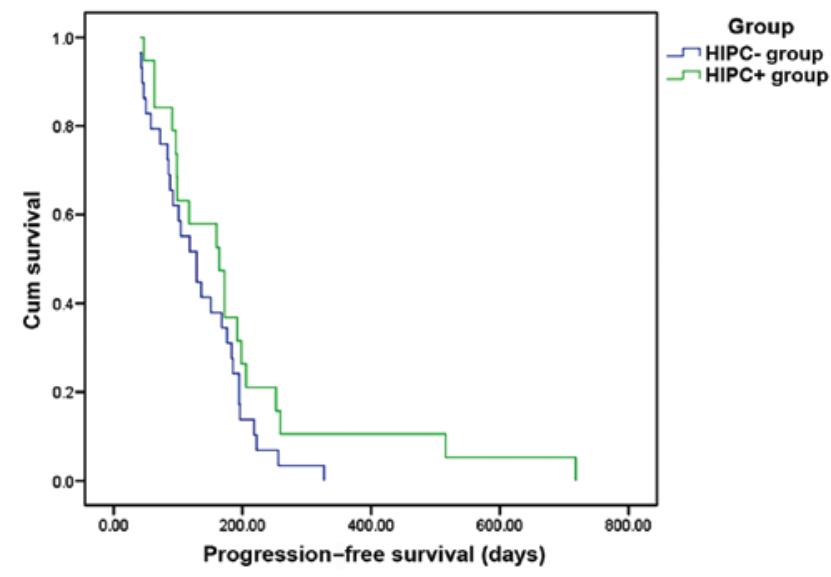

Figure 1. Kaplan-Meier estimates of progression-free survival (PFS) in the HIPC+ and HIPC- group. The median PFS was 164 days in the HIPC+ and 129 days in the HIPC- group. HIPEC, hyperthermic intraperitoneal chemotherapy; cum, cumulative.

(Hangzhou, China) from 2008-1-1 through to 2014-12-31 were retrospectively analyzed (Table I). Patients eligible for this study had histologically confirmed GC with PM, which means positive peritoneal cytology. The patients had been treated with mono- or combination chemotherapy, which was found to be effective in GC, including S-1, S-1 + cisplatin (SP),

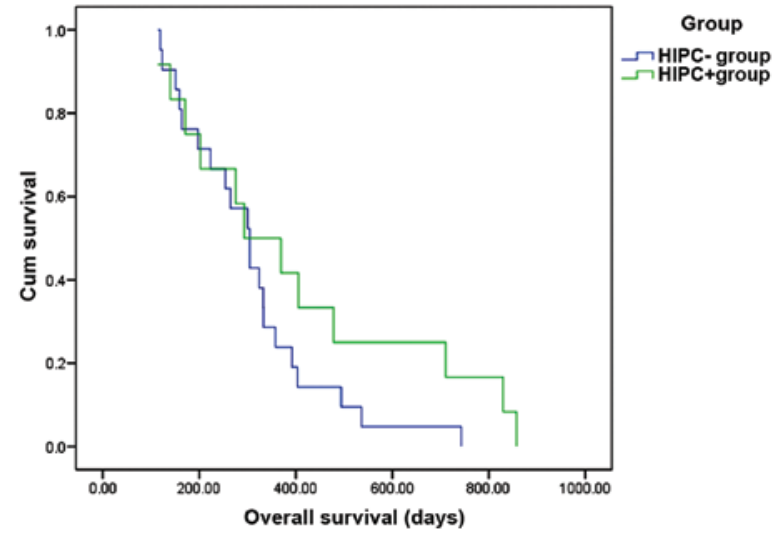

Figure 2. Kaplan-Meier estimates of overall survival (OS) in the HIPC+ and HIPC- group. The median OS was 494 days in the HIPC+ and 223 days in the HIPC- group $(\mathrm{P}=0.178)$. The 1 -year survival rate was $41.7 \%$ in the $\mathrm{HIPC}+$ and $23.8 \%$ in the HIPC- group $(\mathrm{P}<0.001)$. HIPEC, hyperthermic intraperitoneal chemotherapy; cum, cumulative.

S-1 + oxaliplatin (SOX), capecitabine + oxaliplatin (XELOX), capecitabine + docetaxel (XT), oxaliplatin + 5-fluorouracil + folinic acid (FOLFOX), docetaxel + cisplatin (DP), Doxetaxel + S1 (DS), Doxetaxel + Xeloda (capecitabine) (DX) and irinotecan (CPT-11). Finally, a total of 33 patients had complete survival data, including 12 in the HIPEC+ and 21 in the HIPEC- group. 


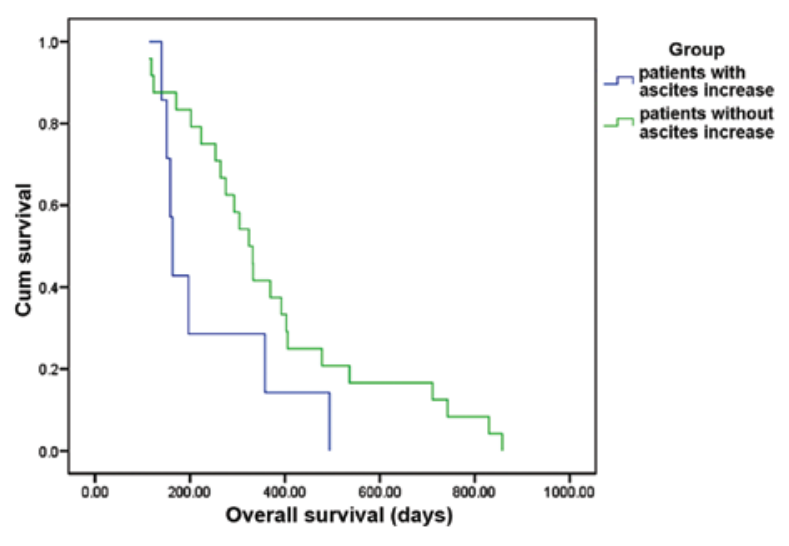

Figure 3. Kaplan-Meier estimates of overall survival (OS) in different groups according to the ascites control status (the OS in patients with ascites disappear + decrease + stable appeared to be better compared with that in patients with ascites increase, but the difference was not statistically significant $(\mathrm{P}=0.08)$.

Table II. Correlation analysis between baseline characteristics and overall survival $(n=33)$.

\begin{tabular}{lc}
\hline Variables & P-value \\
\hline Gender & 0.102 \\
Age & 0.967 \\
Primary site & 0.731 \\
Pathology & 0.910 \\
Radical operation & 0.178 \\
\hline
\end{tabular}

The present study was approved by the Ethics Committee and Institutional Review Board of the Zhejiang Cancer Hospital and was conducted in compliance with the ethical principles of the Declaration of Helsinki.

Treatment. Among the 54 patients, 23 received systemic chemotherapy combined with HIPEC (HIPEC+ group) and 31 patients received systemic chemotherapy alone (HIPEC-group). The systemic chemotherapy was a mono- or combination therapy, which was found to be effective in GC, including S1, SP, SOX, XELOX, XT, FOLFOX, DP, DS, DX and CPT-11. In the HIPEC+ group, the patients were administered cisplatin (CDDP, $50 \mathrm{mg} / \mathrm{m}^{2}$ ) through intraperitoneal perfusion during chemotherapy and then abdominal hyperthermia was applied in vitro. The doses of chemotherapy and their adjustments were made according to each patient's status.

Adverse effects. Toxicity was measured using the National Cancer Institute Common Toxicity Criteria version 2.0 toxicity scales (19). Grade 3-4 toxicity was recorded according to the medical records.

Assessment and statistics. Response was evaluated every two cycles of treatment using the Response Evaluation Criteria in Solid Tumors (20). The patients were divided into 4 categories according to the changes in ascites, including disappear, decrease, stable and increase. Survival time was analyzed

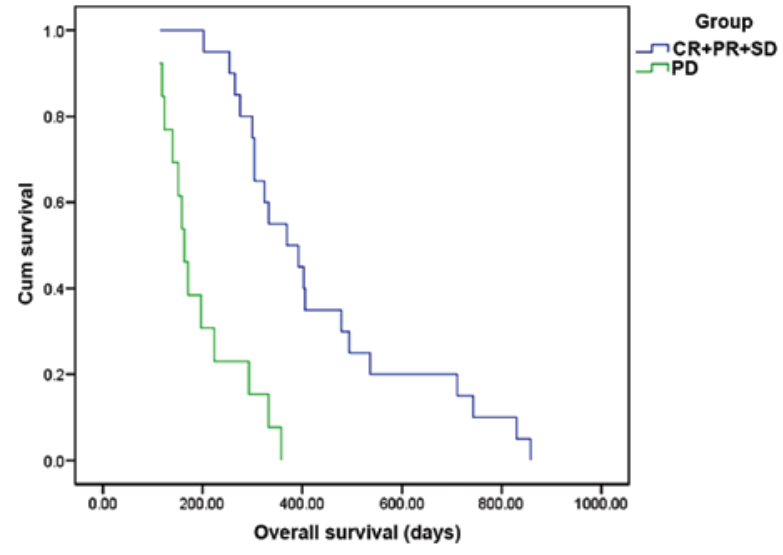

Figure 4. Kaplan-Meier estimates of overall survival (OS) in different groups according to the disease control status. Patients with controlled disease [complete response $(\mathrm{CR})+$ partial response $(\mathrm{PR})+$ stable disease $(\mathrm{SD})]$, had better $\mathrm{OS}$ compared with patients with progressive disease $(\mathrm{PD} ; \mathrm{P}<0.01)$.

with the Kaplan-Meier method using the SPSS software, version 15.0 (SPSS, Inc., Chicago, IL, USA).

\section{Results}

Efficacy. The patients were divided into 4 categories according to the changes of ascites, namely disappear, decrease, stable and increase. In the 54 patients, the disappear + decrease rate in the HIPEC+ group was $82.60 \%$, which was superior to that in the HIPEC- group $(54.80 \%$; $\mathrm{P}=0.043)$. The disappear + decrease + stable rate was $95.70 \%$ in the HIPEC+ group and $74.20 \%$ in the HIPEC- group $(\mathrm{P}=0.062)$. The disease control rate was $65.22 \%$ in the HIPEC+ group and $58.06 \%$ in the HIPEC- group $(\mathrm{P}=0.594)$. Therefore, HIPEC may effectively control ascites in advanced GC.

Of the 33 patients with complete survival data available, including 12 from the HIPEC+ and 21 from the HIPEC- group, further analysis revealed that the median progression-free survival (PFS) was 164 days in the HIPEC+ group and 129 days in the HIPEC- group $(\mathrm{P}=0.158$; Fig. 1$)$. The median $\mathrm{OS}$ was 494 days in the HIPEC+ and 223 days in the HIPEC- group $(\mathrm{P}=0.178)$, and the 1 -year survival rate was 41.7 and $23.8 \%$, respectively $(\mathrm{P}<0.001$; Fig. 2$)$. In patients with ascites disappear + decrease + stable, the OS appeared to be better compared with that in patients with ascites increase, but the difference was not statistically significant $(\mathrm{P}=0.08$; Fig. 3$)$. Patients with controlled disease [complete response $(\mathrm{CR})+$ partial response $(\mathrm{PR})+$ stable disease (SD)], may have a better OS compared with patients with progressive disease $(\mathrm{P}<0.001$; Fig. 4). Other factors, such as gender, age, primary tumor site, pathology, and radical surgery, exhibited no obvious correlation with OS (Table II).

Toxicity. All 54 patients were assessed for treatment safety, and no grade 3 or 4 adverse events or treatment-related deaths were recorded.

\section{Discussion}

HIPEC has been proven to be effective in the treatment of PM in ovarian, appendiceal and colorectal cancer (21-24), but its 
Table III. Clinical results of GC patients with PM treated with CRS and HIPEC.

\begin{tabular}{|c|c|c|c|c|c|c|c|c|}
\hline \multirow[b]{2}{*}{ Authors (Refs.) } & \multirow{2}{*}{$\begin{array}{c}\text { Publication } \\
\text { year }\end{array}$} & \multirow{2}{*}{ Country } & \multirow{2}{*}{$\begin{array}{l}\text { No. of } \\
\text { patients }\end{array}$} & \multirow{2}{*}{$\begin{array}{l}\text { Drug } \\
\text { regimen }\end{array}$} & \multicolumn{2}{|c|}{ Temperature $\left({ }^{\circ} \mathrm{C}\right)$} & \multirow{2}{*}{$\begin{array}{l}\text { Median survival } \\
\text { (months) }\end{array}$} & \multirow{2}{*}{$\begin{array}{l}\text { 1-year } \\
\text { survival } \\
\text { rate }(\%)\end{array}$} \\
\hline & & & & & Inflow & Outflow & & \\
\hline Fujimoto et al (25) & 1997 & Japan & 48 & MMC & $44.5-45.0$ & $43-44$ & & 54.0 \\
\hline Beaujard et al (26) & 2000 & France & 42 & MMC & $46-49$ & $36-43$ & & 48.0 \\
\hline Mussa et al (27) & 2001 & Italy & 7 & MMC & & $42-43$ & & 68.0 \\
\hline Yonemura et al (28) & 2005 & Japan & 107 & $\begin{array}{c}\text { MMC, } \\
\text { CDDP, ETP }\end{array}$ & & $42-43$ & 11.5 & 35.5 \\
\hline Glehen et al (29) & 2010 & France & 159 & $\begin{array}{l}\text { MMC, CDDP } \\
\text { or OXIRI, 5-FU }\end{array}$ & & $40-43$ & 9.2 & 43.0 \\
\hline Yang et al (30) & 2010 & China & 21 & $\begin{array}{l}\text { Hydroxycamptochecin } \\
\text { or CDDP, MMC }\end{array}$ & & 43 & & 50.0 \\
\hline Yang et al (31) & 2011 & China & 34 & $\mathrm{MMC}, \mathrm{CDDP}$ & & 43 & 11.0 & 41.2 \\
\hline Magge et al (32) & 2013 & USA & 23 & $\mathrm{MMC}, \mathrm{CDDP}$ & & 42 & 9.5 & 50.0 \\
\hline
\end{tabular}

GC, gastric cancer; PM, peritoneal metastasis; CRS, cytoreductive surgery; HIPEC, heated intraoperative intraperitoneal chemotherapy; CDDP, cisplatin; MMC, mitomycin; PT, paclitaxel; ETP, etoposide; OX, oxaliplantin; IRI, irinotecan; 5-FU, 5-flourouracil.

role in GC with PM has not been established. The present study retrospectively investigated GC patients with PM from 2008-1-1 through to 2014-12-31 at the Zhejiang Cancer Hospital. The results demonstrated that the disappear + decrease rate in the HIPEC+ group was better compared with that in the HIPECgroup, which means that HIPEC may effectively control ascites and significantly improve the quality of life in GC patients with PM. This may be a major step in the fight against cancer.

In patients with complete survival data, the survival curves of the two groups were separated in Kaplan-Meier estimates of PFS and OS: The 1-year survival rate of HIPEC+ group was significantly higher compared with that of the HIPEC- group. Recent studies on GC patients with PM treated with cytoreductive surgery (CRS) and HIPEC reported 1-year OS rates ranging from 35.5 to $50 \%$ (Table III) (25-32). Our results are similar, but considered encouraging, due to the avoidance of surgical trauma. However, the difference in PFS and OS between the two groups was not statistically significant. This may be attributed to the small number of cases, which suggests that further studies with larger samples are required to confirm our data.

Further analysis in our study demonstrated that in patients with ascites disappear + decrease + similar, the OS appeared to be better compared with that in patients with ascites increase; the survival curves of the two groups were separated, but the difference was not statistically significant. In addition, patients with controlled disease $(\mathrm{CR}+\mathrm{PR}+\mathrm{SD})$, may have a better OS compared with patients with progressive disease $(\mathrm{P}<0.001)$. These results indicate that short-term remission of the disease, including control of ascites, is associated with good prognosis. Further research on the association between short-term remission and the prognosis of advanced GC is planned in the near future.

Toxicity is also a major concern. As HIPEC has the advantage of increasing the concentration of chemotherapeutic agents locally administered into the peritoneum with fewer systemic side effects compared with systemic chemotherapy, it may be hypothesized that the combination of heat and drug toxicity may lead to more complications, such as anastomotic leakage, intra-abdominal abscess, wound infection, pulmonary edema and adult respiratory distress syndrome (25). In this study, none of the abovementioned serious adverse effects were observed, and no other serious unacceptable adverse effects or treatment-related deaths were reported.

In summary, this study demonstrated the effectiveness and safety of HIPEC combined with systemic chemotherapy for advanced GC with peritoneal dissemination; however, large multicenter prospective randomized controlled studies and pharmacokinetic analysis are required to confirm our findings.

\section{Acknowledgements}

The present study was supported by the General Research Program of Medical Health in Zhejiang Province (grant nos. 2011KYA032, 2014KYB039 and 2016KYB036), the Scientific Research Fund Project of Integrated Chinese and Western Medicine Institute in Zhejiang province (grant no. 2014LYK021), the Science and Technology in Zhejiang Province Chinese Medicine Program (grant nos. 2012ZA101 and 2016ZA038), the Hangzhou City Science and Technology Project Planning Guide (Social Development) (grant no. 20130733Q15) and the Hangzhou City Health Science and Technology Project (grant no. 2013A43).

\section{References}

1. Brigand C, Arvieux C, Gilly FN and Glehen O: Treatment of peritoneal carcinomatosis in gastric cancers. Dig Dis 22: 366-373, 2004.

2. Isobe Y,Nashimoto A, Akazawa K, Oda I,Hayashi K, Miyashiro I, Katai H, Tsujitani S, Kodera Y, Seto Y and Kaminishi M: Gastric cancer treatment in Japan: 2008 annual report of the JGCA Nationwide Registry. Gastric Cancer 14: 301-316, 2011. 
3. Yonemura Y, Endou Y, Sasaki T, Hirano M, Mizumoto A Matsuda T, Takao N, Ichinose M, Miura M and Li Y: Surgical treatment for peritoneal carcinomatosis from gastric cancer. Eur J Surg Oncol 36: 1131-1138, 2010

4. Kodera Y, Ito Y, Ito S, Ohashi N, Mochizuki Y, Yamamura Y, Koike M, Fujiwara M, Nakanishi H and Nakao A: Intraperitoneal paclitaxel: A possible impact of regional delivery for prevention of peritoneal carcinomatosis in patients with gastric carcinoma. Hepatogastroenterology 54: 960-963, 2007.

5. Ishigami H, Kitayama J, Otani K, Kamei T, Soma D, Miyato H, Yamashita H, Hidemura A, Kaisaki S and Nagawa H: Phase I pharmacokinetic study of weekly intravenous and intraperitoneal paclitaxel combined with S-1 for advanced gastric cancer. Oncology 76: 311-314, 2009.

6. Ishigami H, Kitayama J, Kaisaki S, Hidemura A, Kato M, Otani K, Kamei T, Soma D, Miyato H, Yamashita $H$ and Nagawa H: Phase II study of weekly intravenous and intraperitoneal paclitaxel combined with S-1 for advanced gastric cancer with peritoneal metastasis. Ann Oncol 21: 67-70, 2010.

7. Ishigami H, Kitayama J, Kaisaki S, Yamaguchi H, Yamashita $H$ Emoto $\mathrm{S}$ and Nagawa H: Phase I study of biweekly intravenous paclitaxel plus intraperitoneal cisplatin and paclitaxel for gastric cancer with peritoneal metastasis. Oncology 79: 269-272, 2010.

8. Matharu G, Tucker O and Alderson D: Systematic review of intraperitoneal chemotherapy for gastric cancer. Br J Surg 98 $1225-1235,2011$.

9. Yonemura Y, Ninomiya I, Kaji M, Sugiyama K, Fujimura T, Sawa T, Katayama K, Tanaka S, Hirono Y, Miwa K, et al: Prophylaxis with intraoperative chemohyperthermia against peritoneal recurrence of serosal invasion-positive gastric cancer. World J Surg 19: 450-454; discussion 455, 1995.

10. Dedrick RL: Theoretical and experimental bases of intraperitoneal chemotherapy. Semin Oncol 12 (3 Suppl 4): S1-S6, 1985.

11. Shimada T, Nomura M, Yokogawa K, Endo Y, Sasaki T, Miyamoto K and Yonemura Y: Pharmacokinetic advantage of intraperitoneal injection of docetaxel in the treatment for peritoneal dissemination of cancer in mice. J Pharm Pharmacol 57: 177-181, 2005.

12. Speyer JL, Sugarbaker PH, Collins JM, Dedrick RL, Klecker RW Jr and Myers CE: Portal levels and hepatic clearance of 5-fluorouracil after intraperitoneal administration in humans. Cancer Res 41: 1916-1922, 1981.

13. Landry J, Tepper JE, Wood WC, Moulton EO, Koerner F and Sullinger J: Patterns of failure following curative resection of gastric carcinoma. Int J Radiat Oncol Biol Phys 19: 1357-1362, 1990.

14. Shiu MH and Fortner JG: Intraperitoneal hyperthermic treatment of implanted peritoneal cancer in rats. Cancer Res 40: 4081-4084, 1980.

15. Zhao C, Dai C and Chen X: Whole-body hyperthermia combined with hyperthermic intraperitoneal chemotherapy for the treatment of stage IV advanced gastric cancer. Int J Hyperthermia 28: 735-741, 2012.

16. Sun J, Song Y, Wang Z, Gao P, Chen X, Xu Y, Liang J and Xu H: Benefits of hyperthermic intraperitoneal chemotherapy for patients with serosal invasion in gastric cancer: A meta-analysis of the randomized controlled trials. BMC Cancer 12: 526, 2012.

17. Esquivel J, Elias D, Baratti D, Kusamura S and Deraco M: Consensus statement on the loco regional treatment of colorectal cancer with peritoneal dissemination. J Surg Oncol 98: 263-267, 2008.

18. Gómez Portilla A: Peritoneal carcinomatosis. Ten years of applying the new combined triple therapy. Personal experience. Cir Esp 82: 346-351, 2007 (In Spanish).
19. Shimizu T and Saijo N: Common toxicity criteria: version 2.0 , an improved reference for grading the adverse reaction of cancer treatment. Nihon Rinsho 61: 937-942, 2003.

20. Eisenhauer EA, Therasse P, Bogaerts J, Schwartz LH, Sargent D, Ford R, Dancey J, Arbuck S, Gwyther S, Mooney M, et al: New response evaluation criteria in solid tumours: Revised RECIST guideline (version 1.1). Eur J Cancer 45: 228-247, 2009.

21. Alberts DS, Markman M, Armstrong D, Rothenberg ML, Muggia F and Howell SB: Intraperitoneal therapy for stage III ovarian cancer: A therapy whose time has come! J Clin Oncol 20: 3944-3946, 2002.

22. Jaaback K, Johnson N and Lawrie TA: Intraperitoneal chemotherapy for the initial management of primary epithelial ovarian cancer. Cochrane Database Syst Rev CD005340, 2011.

23. Verwaal VJ, van Ruth S, de Bree E, van Slooten GW, van Tinteren H, Boot $\mathrm{H}$ and Zoetmulder FA: Randomized trial of cytoreduction and hyperthermic intraperitoneal chemotherapy versus systemic chemotherapy and palliative surgery in patients with peritoneal carcinomatosis of colorectal cancer. J Clin Oncol 21: 3737-3743, 2003.

24. Sugarbaker PH: New standard of care for appendiceal epithelia neoplasms and pseudomyxoma peritonei syndrome? Lancet Oncol 7: 69-76, 2006

25. Fujimoto S, Takahashi M, Mutou T, Kobayashi K, Toyosawa T, Isawa $\mathrm{E}$, Sumida $\mathrm{M}$ and Ohkubo H: Improved mortality rate of gastric carcinoma patients with peritoneal carcinomatosis treated with intraperitoneal hyperthermic chemoperfusion combined with surgery. Cancer 79: 884-891, 1997.

26. Beaujard AC, Glehen O, Caillot JL, Francois Y, Bienvenu J, Panteix G, Garbit F, Grandclément E, Vignal J and Gilly FN: Intraperitoneal chemohyperthermia with mitomycin $\mathrm{C}$ for digestive tract cancer patients with peritoneal carcinomatosis. Cancer 88: 2512-2519, 2000.

27. Mussa A, Sandrucci S and Zanon C: Intraoperative chemohyperthermia for advanced gastric cancer: A new procedure with closed abdomen and previously constructed anastomosis. Tumori 87 (Suppl): S18-S20, 2001.

28. Yonemura Y, Kawamura T, Bandou E, Takahashi S, Sawa T and Matsuki N: Treatment of peritoneal dissemination from gastric cancer by peritonectomy and chemohyperthermic peritoneal perfusion. Br J Surg 92: 370-375, 2005.

29. Glehen O, Gilly FN, Arvieux C, Cotte E, Boutitie F, Mansvelt B, Bereder JM, Lorimier G, Quenet F and Elias D; Association Française de Chirurgie: Peritoneal carcinomatosis from gastric cancer: A multi-institutional study of 159 patients treated by cytoreductive surgery combined with perioperative intraperitoneal chemotherapy. Ann Surg Oncol 17: 2370-2377, 2010.

30. Yang XJ, Li Y and Yonemura Y: Cytoreductive surgery plus hyperthermic intraperitoneal chemotherapy to treat gastric cancer with ascites and/or peritoneal carcinomatosis: Results from a Chinese center. J Surg Oncol 101: 457-464, 2010.

31. Yang XJ, Huang CQ, Suo T, Mei LJ, Yang GL, Cheng FL, Zhou YF, Xiong B, Yonemura Y and Li Y: Cytoreductive surgery and hyperthermic intraperitoneal chemotherapy improves survival of patients with peritoneal carcinomatosis from gastric cancer: Final results of a phase III randomized clinical trial. Ann Surg Oncol 18: 1575-1581, 2011.

32. Magge D, Zenati M, Mavanur A, Winer J, Ramalingam L, Jones H, Zureikat A, Holtzman M, Lee K, Ahrendt S, et al: Aggressive locoregional surgical therapy for gastric peritoneal carcinomatosis. Ann Surg Oncol 21: 1448-1455, 2014. 